Dokuz Eylül University, adem.celik@deu.edu.tr, İzmir-Turkey

DOI $\quad$ http://dx.doi.org/10.12739/NWSA.2018.13.4.3A0086

ORCID ID $0000-001-9007-2937$

CORRESPONDING AUTHOR Adem Çelik

\title{
ADİ DİFERANSIYYEL DENKLEMLER İÇİN BİR NOT: K-KARE DİFERANSİYEL DENKLEMLER VE ÇÖZÜMLERI
}

Öz

Bu çalışmada, adi diferansiyel denklemler için "k-kare diferansiyel denklemler" tanımlanmıştır. Bu denklemlerin genel çözümü araştırılmıştır. Buna bağlı olarak, bazı lineer tip, Cauchy-Euler tipi, Legendre tipi ve lineer olmayan tip diferansiyel denklemler için uygulamalar yapılmışır. Ayrıca, kompleks analize genişletme yapılmıştır.

Anahtar Kelimeler: Matematik Analiz, Adi diferansiyel denklem, Lineer diferansiyel denklem, lineer olmayan Diferansiyel denklem, k-Katlı İntegral

\section{A NOTE FOR ORDINARY DIFFERENTIAL EQUATIONS: K-SQUARE DIFFERENTIAI EQUATIONS AND SOLUTIONS}

\section{ABSRACT}

In this study, "k-square differential equations" for ordinary differential equations are defined. The general solution of these equations is investigated. Accordingly, somelinear equation types, Cauchy-Euler type, Legendre type and nonlinear equations have been applied. In addition, the complex analysis expansions were developed.

Keywords: Mathematical Analysis, Ordinary Differential Equations, Linear Differential Equations, Nonlinear Differential Equations, k-fold Integral 


\section{GİRIŞ (INTRODUCTION)}

$y^{(k)}=f(x)$ diferansiyel denkleminin genel çözümü bulmak isteyelim: $y^{(k)}=\left(y^{(k-1)}\right)^{\prime}$ olduğunu göz önüne alırsak, $y^{(k-1)}=\int_{x_{0}}^{x} f(x) d x+C_{1}$ olur. Burada $x_{0} x$ 'in bir sabit değeri ve $C_{1}$ integral sabitidir. Bunu da entegre edersek, $\quad y^{(k-2)}=\int_{x_{0}}^{x}\left(\int_{x_{0}}^{x} f(x) d x\right) d x+C_{1}\left(x-x_{0}\right)+C_{2}$ olur. Böyle devam ederek $(k$ integral işleminden sonra) integralin tamamı, $\mathrm{y}=\int_{\mathrm{x}_{0}}^{\mathrm{x}} \ldots \int_{\mathrm{x}_{0}}^{\mathrm{x}} f(x) d x \ldots d x+$ $\frac{\mathrm{C}_{1}\left(\mathrm{x}-\mathrm{x}_{0}\right)^{\mathrm{k}-1}}{(\mathrm{k}-1) !}+\frac{\mathrm{C}_{2}\left(\mathrm{x}-\mathrm{x}_{0}\right)^{\mathrm{k}-2}}{(\mathrm{k}-2) !}+\cdots+\mathrm{C}_{\mathrm{k}}$ formunda olur [4]. Bu diferansiyel denklemler n tane birinci mertebe adi diferansiyel denklemin bir sistemine dönüştürülebilir [3] $. \frac{d^{k} y}{d x^{k}}=f(x)$ tipi diferansiyel denklemlerde art arda integral alma işlemiyle sonuca gideriz [6]. $a_{0} x^{n} y^{(n)}+a_{1} x^{n-1} y^{(n-1)}+\ldots$ $+a_{n} y=b(x)$ tipindeki diferansiyel denklemlere Cauchy-Euler diferansiyel denklemleri denir. Burada $a_{0}, a_{1}, \ldots, a_{n}$ ler reel sabitlerdir. Uygun bir dönüşümle bu denklemler sabit katsayılı bir lineer denkleme dönüşür. Her bir terimi $(b x+c)^{k} y^{(k)}$ ifadesinin bir sabitle çarpımı şeklinde olan $a_{0}(b x+c)^{k} y^{(k)}+a_{1}(b x+c)^{k-1} y^{(k-1)}+\ldots+a_{k-1}(b x+c) y^{\prime}+a_{k} y=b(x)$ tipindeki

diferansiyel denklemlere Legendre denklemi denir. Bu denklemler de uygun bir dönüşümle Cauchy-Euler denklemine dönüşürler [6, 7 and 8]. Homojen olmayan $y^{\prime \prime}+P(x) y^{\prime}+Q(x) y=f(x)$ diferansiyel denkleminde $f(x)$ çok sayıda terimden oluşsun. Varsayalım ki $f(x)=f_{1}(x)+f_{2}(x)+\cdots+f_{n}(x)$ dir. Yani $f$ fonksiyonu $\mathrm{n}$ adet $f_{i}(i=1,2, \ldots, n)$ fonksiyonlarının toplamı olarak ifade edilmiş olsun. Bu durumdaki denklemin $y_{\ddot{0}}(x)$ özel çözümünü arayalım. Her bir $f_{i}$ fonksiyonu için özel çözümü $y_{\ddot{o}_{i}}(x)$ ile gösterelim. Bu halde $y_{\ddot{0}_{1}}(x)+y_{\ddot{0}_{2}}(x)+\cdots+y_{\ddot{o}_{n}}(x)$ toplamı verilen denklemin bir özel çözümüdür. Yani $y_{\ddot{o}}(x)=y_{\ddot{0}_{1}}(x)+y_{\ddot{0}_{2}}(x)+\cdots+y_{\ddot{o}_{n}}(x)$ dır. Bu ilke süperpozisyon ilkesi olarak isimlendirilir [7]. z kompleks değişken, $\mathbb{C}$ kompleks sayılar cismi ve $\mathbf{A} \subset \mathbb{C}$ bir bölge olmak üzere, $f: A \rightarrow \mathbb{C}, w=f(z)$ ve $F: A \rightarrow \mathbb{C}, w=F(z)$ fonksiyonları A bölgesinde analitik olsun. Eğer $F^{\prime}(z)=$ $f(z)$ ise, $F$ fonksiyonuna $f$ fonksiyonunun belirsiz integralidir denir ve $C \in \mathbb{C}$ (belirsiz integral sabiti) olmak üzere, $\int f(z) d z=F(z)+C$ biçiminde yazılır [1, 2 ve 5].

\section{2. ÇALIŞMANIN ÖNEMI (RESEARCH SIGNIFICANSE)}

Bu makalede, adi diferansiyel denklemler için "k-kare diferansiyel denklemler" tanımı yapılmıştır. Bu denklemlerin genel çözümleri verilmiş ve ispatlanmıştır. k-kare diferansiyel denklemler $y^{(k)}=f(x)$ diferansiyel denkleminden daha genel diferansiyel denklemlerdir.

\section{ANALITTIK ÇALIŞMA (ANALYTICAL STUDY)}

$\mathrm{Bu}$ makale teorik tabanlı bir analitik araştırma makalesidir. Bu ispat tekniği, hipotez-hüküm çerçevesinde şekillenir [9].

\section{K-KARE DİFERANSIYYEL DENKLEMLER (K-SQUARE DIFFERENTIAL EQUATIONS)}

$k=1,2, \ldots n$ ve $m \in \mathbb{Q}(m \neq 0)$ olsun.

- Tanım $1: g, h$ fonksiyonları bir $I \subset \mathbb{R}$ aralığında analitik, $y^{m}$ fonksiyonu aynı aralıkta sürekli ve $k(k=1,2, \ldots, n)$ mertebeden türevli olmak üzere, her $x \in \mathrm{I}$ için $g(x) \neq 0, y(x) \neq 0$ olsun. Eğer $F\left(x, y, y^{\prime}, y^{\prime \prime} \ldots, y^{(k)}\right)=0$ diferansiyel denklemi, $\left(y^{m} \cdot h(x)\right)^{(k)}-g(x)=0$ $\left(y^{m}+h(x)\right)^{(k)}-g(x)=0$ 
formlarından biri biçiminde yazılabilirse bir "k-kare diferansiyel denklemdir" denir.

- Teorem 1. $F\left(x, y, y^{\prime}, y^{\prime \prime} \ldots, y^{(k)}\right)=0$ diferansiyel denklemi verilsin. Bu denklem tipinde bir $k$-kare diferansiyel denklem ise, bunun genel çözümü;

$y^{m}=\frac{1}{h(x)} \iiint \ldots \int g(x)(d x)^{k}$

fonksiyonudur.

(2) tipinde bir $k$-kare diferansiyel denklem ise, bunun genel çözümü $y^{m}=\iiint \ldots \int g(x)(d x)^{k}-h(x)$

Fonksiyonudur.

- İspat: i) $\iiint \ldots \int g(x)(d x)^{k}=y^{m} \cdot h(x) \quad$ eşitliğini ele alalım. Bu eşitliğin her iki yanının $k(k=1,2, \ldots n)$ mertebeden türevi alınırsa, integral hesabin temel teoreminden

$\left(y^{m} \cdot h(x)\right)^{(k)}=g(x)$ ya da $(y \cdot g(x))^{(k)}-g(x)=0$ bulunur. Bu diferansiyel denklemi çözmek isteyelim. Başka bir deyişle (1) denklemini sağlayan $y\left(y^{m}\right)$ 'yi bulalım. (3) denklemi buna izin verir ve bundan (1) bulunur. Şimdi $y\left(y^{m}\right)$ 'nin (1) denkleminin genel çözümü olduğunu gösterelim. Bunun için (1) denkleminin k-1 katlı belirsiz integralini alalım, $C_{1}, C_{2}, C_{3}, \ldots, C_{k-1}, C_{k}$ integral sabitleri olmak üzere

$y^{m}=\frac{1}{h(x)}\left[\int H_{k-1}(x) \cdot d x+C_{k}\right]+\frac{1}{h(x)}\left[C_{k-1} \cdot \frac{x^{k-1}}{(k-1) !}+C_{k-2} \cdot \frac{x^{k-2}}{(k-2) !}+\cdots+C_{1} \cdot x\right] \quad$ veya

$y^{m}=\frac{1}{h(x)}\left[\int H_{k-1}(x) \cdot d x\right]+\frac{1}{h(x)}\left[C_{k}+C_{k-1} \cdot \frac{x^{k-1}}{(k-1) !}+C_{k-2} \cdot \frac{x^{k-2}}{(k-2) !}+\cdots+C_{1} \cdot x\right]$

biçiminde bir fonksiyon elde edilir. Burada $H_{k-1}$ fonksiyonu, $\mathrm{g}(\mathrm{x})^{\prime}$ in alınan $k-1$ katlı integraller sonunda elde edilen fonksiyondur. (5) de $\left\{x^{k-1}, x^{k-2}, \ldots, x, 1\right\}$ kümesi lineer bağımsızdır. 0 zaman

$\left\{\frac{x^{k-1}}{h(x)}, \frac{x^{k-2}}{h(x)}, \ldots, \frac{x}{h(x)}, \frac{1}{h(x)}\right\}$ de lineer bağımsız olur. Şimdi, (5)'in $y^{m}=$ $\frac{1}{h(x)}\left[\int H_{k-1}(x) \cdot d x\right]$ parçasını ele alalım. Bu eşitlikten $y^{m} \cdot h(x)=$ $\int H_{k-1}(x) \cdot d x$ yazılır. Bunun her iki tarafının $k$ mertebeden türevini alalım, $\left(y^{m} \cdot h(x)\right)^{(k)}=g(x)$ ya da $\left(y^{m} \cdot h(x)\right)^{(k)}-g(x)=0$ elde edilir. Yani bu parça da (1) denklemini sağlar. Yani bir çözümdür. Böylece (2) deki $y$ fonksiyonu $k$ tane keyfi sabit içerdiğinden ( $1^{\prime}$ in genel çözümüdür.

ii) $\iiint \ldots \int g(x)(d x)^{k}=y^{m}+h(x)$ eşitliğini ele alalım. $\left.\dot{I}\right)$ şıkkındaki ispat yolunu izlersek, $\left(y^{m}+h(x)\right)^{(k)}=g(x)$ ya da $\left(y^{m}+h(x)\right)^{(k)}-g(x)=0$ buluruz. Aynı şekilde $C_{1}, C_{2}, C_{3}, \ldots, C_{k-1}, C_{k}$ integral sabitler olmak üzere

$y^{m}+h(x)=\left[\int H_{k-1}(x) \cdot d x+C_{k}\right]+\left[C_{k-1} \cdot \frac{x^{k-1}}{(k-1) !}+C_{k-2} \cdot \frac{x^{k-2}}{(k-2) !}+\cdots+C_{1} \cdot x\right] \quad$ veya

$y^{m}=\left[\int H_{k-1}(x) \cdot d x\right]-h(x)+\left[C_{k}+C_{k-1} \cdot \frac{x^{k-1}}{(k-1) !}+C_{k-2} \cdot \frac{x^{k-2}}{(k-2) !}+\cdots+C_{1} \cdot x\right]$

biçiminde bir fonksiyon elde edilir. (6) da gene $H_{k-1}$ fonksiyonu, $g(x)^{\prime}$ in alınan k-1 katlı integraller sonunda elde edilen fonksiyondur. Sonra üstteki denklemin

$y^{m}=\left[\int H_{k-1}(x) \cdot d x\right]-h(x)$

parçasını ele alırız ve İ) deki tartışmayı yaparız. Teorem 1'in ışığında aşağıdaki sonuçları verebiliriz.

Sonuç 1. Eğer (1) denkleminde $g(x)=0$ ise $\left(y^{m} \cdot h(x)\right)^{(k)}=0$ ikinci yansız (homojen) k- kare diferansiyel denklemleri elde edilir. Bu diferansiyel denklemlerin genel çözümü, $\int 0 d x=C($ sabit $)$ olduğuna göre, 
$y^{m}=\frac{1}{h(x)} \iiint \ldots \int 0(d x)^{k}=\frac{1}{h(x)}\left[C_{k}+C_{k-1} \cdot \frac{x^{k-1}}{(k-1) !}+C_{k-2} \cdot \frac{x^{k-2}}{(k-2) !}+\cdots+C_{1} \cdot x\right]$

olur. Burada $C_{1}, C_{2}, C_{3}, \ldots, C_{k-1}, C_{k}$ integral sabitlerdir.

Sonuç 2. Sabit katsayılı bazı k-kare diferansiyel denklemleri (1) de $h(x)=g(x)=e^{a x} \quad(a \neq 0 \quad)$ koyarak $\quad\left(y^{m} \cdot e^{a x}\right)^{(k)}-e^{a x}=0$ elde edilebilir. Bu diferansiyel denklemlerin genel çözümü

$y^{m}=\frac{1}{e^{a x}} \iiint \ldots \int e^{a x}(d x)^{k}$

Biçimin de olur.

Sonuç 3. I) (1) ve (2) de $m=1$ koyarsak, sırasıyla

$(y \cdot h(x))^{(k)}-g(x)=0$

$(y+h(x))^{(k)}-g(x)=0$

(11) lineer diferansiyel denklemleri elde edilir. Bunların genel çözümü, sırasıyla

$y=\frac{1}{h(x)} \iiint \ldots \int g(x)(d x)^{k}$

$y=\iiint \ldots \int g(x)(d x)^{k}-h(x)$

ii) (1) denkleminde $m=1$ ve $h(x)=1$ veya (2) denkleminde $m=1$ ve $h(x)=0$ alalım, $\quad F\left(x, y, y^{\prime}, y^{\prime \prime} \ldots, y^{(k)}\right)=(y)^{(k)}-g(x)=0$ yani $\quad y^{(k)}(x)=g(x)$ diferansiyel denklemi elde edilir. Bu diferansiyel denklemin genel çözümü,

$y=\iiint \ldots \int g(x)(d x)^{k}$

fonksiyonudur [4 ve 6]. Bu çözüm (3) veya (12)'den de elde edilebilir.

\section{UYGULAMALAR (APPLICATIONS)}

Bu kesimde bazı k-kare diferansiyel denklemleri ele alıp genel çözümlerini vereceğiz .

- İkinci yanlı sabit katsayılı ve değişken katsayılı lineer adi diferansiyel denklemlere uygulama:

Önerme 1 .

I) $a \neq 0$ bir reel sayı ve $k \in \mathbb{N}$ olmak üzere, $F\left(x, y, y^{\prime}, \ldots, y^{(k)}\right)=$

$a^{k} y+\frac{k}{1 !} a^{k-1} y^{\prime}+\frac{k \cdot(k-1)}{2 !} a^{k-2} y^{\prime \prime}+\frac{k \cdot(k-1) \cdot(k-2)}{3 !} a^{k-3} y^{\prime \prime \prime}+\frac{k(k-1)(k-2)(k-3)}{4 !} a^{k-4} y^{(4)}+\ldots$

$+\frac{k(k-1)(k-2)(k-2) \ldots(k-(k-2))}{(k-1) !} a^{k-(k-1)} y^{(k-1)}+\frac{k(k-1)(k-2) \ldots(k-(k-2))(k-(k-1))}{k !} a^{k-k} y^{(k)}-1=0$

sabit katsayılı, lineer, k-kare diferansiyel denkleminin genel çözümü;

$y=\frac{1}{e^{a x}} \iiint \ldots \int e^{a x}(d x)^{k}$

Veya $y=\frac{1}{a^{k}}+\left[C_{k}+C_{k-1} \cdot \frac{x^{k-1}}{(k-1) !}+C_{k-2} \cdot \frac{x^{k-2}}{(k-2) !}+\cdots+C_{1} \cdot x\right] \cdot e^{-a x}$ fonksiyonudur.

ii) $g(x)$ sabit fonksiyon olmasin ve $k \in \mathbb{N}$ olsun, bu halde,

$F\left(x, y, y^{\prime}, \ldots, y^{(k)}\right)=g(x)^{(k)} y+\frac{k}{1 !} g(x)^{(k-1)} y^{\prime}+\frac{k \cdot(k-1)}{2 !} g(x)^{(k-2)} y^{\prime \prime}+\frac{k \cdot(k-1) \cdot(k-2)}{3 !} g(x)^{(k-3)} y^{\prime \prime \prime}+$ $\frac{k(k-1)(k-2)(k-3)}{4 !} g(x)^{(k-4)} y^{(4)}+\ldots+\frac{k(k-1)(k-2)(k-2) \ldots(k-(k-2))}{(k-1) !} g(x)^{(k-(k-1))} y^{(k-1)}+$ $\frac{k(k-1)(k-2) \ldots(k-(k-2))(k-(k-1))}{k !} g(x)^{(k-k)} y^{(k)}-g(x)=0$

biçiminde ${ }^{k !}$ değişken katsayılı, lineer, k-kare diferansiyel denkleminin genel çözümü,

$y=\frac{1}{g(x)} \iiint \ldots \int g(x)(d x)^{k}$

fonksiyonudur.

iii) $g(x) \neq h(x)$ sabit fonksiyon olmasınlar ve $k \in \mathbb{N}$ olmak üzere $F\left(x, y, y^{\prime}, \ldots, y^{(k)}\right)=$

$h(x)^{(k)} y+\frac{k}{1 !} h(x)^{(k-1)} y^{\prime}+\frac{k \cdot(k-1)}{2 !} h(x)^{(k-2)} y^{\prime \prime}+\frac{k \cdot(k-1) \cdot(k-2)}{3 !} h(x)^{(k-3)} y^{\prime \prime \prime}+$ 
$\frac{k(k-1)(k-2)(k-3)}{4 !} h(x)^{(k-4)} y^{(4)}+\ldots+\frac{k(k-1)(k-2)(k-2) \ldots(k-(k-2))}{(k-1) !} h(x)^{(k-(k-1))} y^{(k-1)}+$ $\frac{k(k-1)(k-2) \ldots(k-(k-2))(k-(k-1))}{k !} h(x)^{(k-k)} y^{(k)}-g(x)=0$

değişken katsayılı, lineer, k-kare diferansiyel denkleminin genel çözümü,

$y=\frac{1}{h(x)} \iiint \ldots \int g(x)(d x)^{k}$

fonksiyonudur.

İspat: i) (10) denkleminde $g(x)=h(x)=e^{a x} \quad(\mathrm{a} \neq 0) \quad$ alalım, $F\left(x, y, y^{\prime}, \ldots, y^{(k)}\right)=\left(y \cdot e^{a x}\right)^{(k)}-e^{a x}=0 \quad$ elde edilir. Buradan da (15 denklemi bulunur ve bu bir k-kare diferansiyel denklemdir. Bunun genel çözümü (16) dır. ii) (10) denkleminde $h(x)=g(x) \neq e^{a x}(\mathrm{a} \neq 0)$ alalım, $\quad F\left(x, y, y^{\prime}, \ldots, y^{(k)}\right)=(y \cdot g(x))^{(k)}-g(x)=0, \quad k$-kare diferansiyel denklemi olur. Bunun da genel çözümü (18) dir. iii)

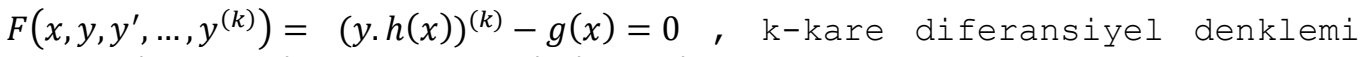
(19) diferansiyel denklemini verir.

- Cauchy-Euler ve Legendre diferansiyel denklemlerine uygulama:

Önerme 2. $\mathrm{k} \geq 1$ bir doğal sayı $x \neq 0 \quad(x>0$ veya $x<0)$ olmak üzere,

i) $k ! y+k \frac{k !}{1 !} x y^{\prime}+\frac{k(k-1)}{2 !} \cdot \frac{k !}{2 !} x^{2} y^{\prime \prime}+\frac{k(k-1)(k-2)}{3 !} \cdot \frac{k !}{3 !} x^{3} y^{\prime \prime \prime}+$ $\frac{k(k-1)(k-2)(k-3)}{4 !} \cdot \frac{k !}{4 !} x^{4} y^{(4)}+\frac{k(k-1)(k-2)(k-3)(k-4)}{5 !} \cdot \frac{k !}{5 !} x^{5} y^{(5)}+\cdots+$

$\frac{k(k-1)(k-2)(k-3) \ldots(k-(k-2))}{(k-1) !} \cdot \frac{k !}{(k-1) !} x^{k-1} y^{(k-1)}+\frac{k(k-1)(k-2)(k-3) \ldots(k-(k-1)}{k !} \cdot \frac{k !}{k !} x^{k} y^{(k)}=g(x)$

Cauchy-Euler tipi diferansiyel denkleminin genel çözümü, $y=\frac{1}{x^{k}} \iiint \ldots \int g(x)(d x)^{k} \quad$ fonksiyonudur.

ii) $\mathrm{k} \geq 1$ bir doğal sayı, $b, c \neq 0$ ve $b x+c \neq 0$ olmak üzere, tüm terimleri (19) dan elde edilebilen

$(b x+c)^{k} y^{(k)}+k^{2} b(b x+c)^{k-1} y^{(k-1)}+\ldots+b^{k} k ! y=g(x)$

Legendre tipi diferansiyel denkleminin genel çözümü,

$y=\frac{1}{(b x+c)^{k}} \iiint \ldots \int g(x)(d x)^{k}$ fonksiyonudur.

İspat: i) Teorem de $m=1$ ve $h(x)=x^{k} \neq 0 \quad(x>0$ veya $x<0)$ alınırsa (21) denklemi elde edilir. ii) Eğer Teorem 1 de $m=1$ için herhangi bir $h$ fonksiyonu yerine $(b x+c)^{k} \neq 0$ polinomu alınırsa (22) formunda Legendre tipi diferansiyel denklem elde edilir.

- İkinci yanlı değişken katsayılı lineer olmayan adi diferansiyel denklemlere uygulamalar: $\mathrm{Bu}$ alt kesimde (1) denklemini sırasıyla k=1,2,3 ve $m \neq 0, m 1, m \in \mathbb{Q}$ için ele alacağız. Aşağıdaki Önerme 3'ü ispatsız olarak vereceğiz.

Önerme 3. (1) denkleminde sırasıyla $k=1, k=2$ ve $k=3$ alalım. Sırasıyla, $m h(x) y^{m-1} \cdot y^{\prime}+h^{\prime}(x) y^{m}=g(x)$

$m h(x) y^{m-1} y^{\prime \prime}+m(m-1) h(x) y^{m-2}\left(y^{\prime}\right)^{2}+2 m h^{\prime}(x) y^{m-1} y^{\prime}+h^{\prime \prime}(x) y^{m}=g(x)$

$m h(x) y^{m-1} y^{\prime \prime \prime}+3 m(m-1) h(x) y^{m-2} y^{\prime} y^{\prime \prime}+3 m h^{\prime}(x) y^{m-1} y^{\prime \prime}+m(m-1)(m-2)$. $h(x) y^{m-3}\left(y^{\prime}\right)^{3}+3 m(m-1) h^{\prime}(x) y^{m-2}\left(y^{\prime}\right)^{2}+3 m h^{\prime \prime}(x) y^{m-1} y^{\prime}+h^{\prime \prime \prime}(x) y^{m}=g(x) \quad$ (25) Lineer olmayan diferansiyel denklemleri elde edilir. (23), (24) ve (23) 'ün genel çözümü de sırasıyla,

$y^{m}=\frac{1}{h(x)} \int g(x) d x$

$y^{m}=\frac{1}{h(x)} \iint g(x)(d x)^{2}$

$y^{m}=\frac{1}{h(x)} \iiint g(x)(d x)^{3}$

olur. 


\section{KOMPLEKS ANALİZE GENİ̧LETME (COMPLEX ANALYSIS EXPANSION)}

bölge olsun.

Tanım 2. $h, g, w$ kompleks değişkenli fonksiyonları bir A bölgesinde analitik olmak üzere, her $z \in A$ için $h(z) \neq 0, w(z) \neq 0$ olsun. Eğer $F\left(z, w, w^{\prime}, w \ldots, w^{(k)}\right)=0 \quad(k=1,2,3, \ldots, n)$ kompleks diferansiyel denklemi, $m \in \mathbb{N}$ ve $m \neq 0$ olmak üzere,

$\left(w^{m} \cdot h(z)\right)^{(k)}-g(z)=0$

$\left(w^{m}+h(z)\right)^{(k)}-g(z)=0$

formlarından biri biçiminde yazılabiliyorsa bir "kompleks k-kare diferansiyel denklemdir" denir.

$g: A \rightarrow \mathbb{C}, \quad w=g(z)$ ve $F: A \rightarrow \mathbb{C}, w=F(z)$ fonksiyonları analitik olmak üzere, $F$ fonksiyonu $g$ fonksiyonunun belirsiz integrali (yani $F^{\prime}=$ g) olsun. Eğer bir kompleks değişkenli fonksiyon bir bölgede analitikse bu fonksiyonun her mertebeden türevi vardır ve bu türev fonksiyonları da aynı bölgede analitiktir [1, 2 ve 5]. Şimdi, belirsiz integral tanımını göz önüne alalım. Fveg kompleks fonksiyonları aynı bir bölgede analitik olmak üzere, eğer $F^{(k)}(z)=g(z) \quad(k=1,2,3, \ldots, n)$ ise $F$ fonksiyonuna $g$ fonksiyonunun $k$ - katlı belirsiz integralidir deriz ve $C \in \mathbb{C}$ ( belirsiz integral sabiti) olmak üzere,

$\iiint \ldots \int g(z)(d z)^{k}=F(z)+C$ yazarız.

Teorem 2. $\quad F\left(z, w, w^{\prime}, w \ldots, w^{(k)}\right)=0 \quad(k=1,2,3, \ldots, n) \quad$ kompleks

diferansiyel denklemi verilsin. Bu denklem;

i) (29) diferansiyel denklemi tipinde ise, genel çözümü

$w^{m}=\frac{1}{h(z)} \iiint \ldots \int g(z)(d z)^{k}$

fonksiyonudur.

İI) (30) diferansiyel denklemi tipinde ise, genel çözümü

$w^{m}=\iiint \ldots \int g(z)(d z)^{k}-h(z)$

fonksiyonudur.

Ispat: Tamamen Teorem $1^{\prime}$ in ispatına benzer olarak yapilır. İlgili fonksiyonların kompleks değişkenli analitik fonksiyonlar olduğu göz önüne alınır. (27) ve (28) de $m=1$ koyarsak, sirasıyla

$(w \cdot h(z))^{(k)}-g(z)=0$

$(w+h(z))^{(k)}-g(z)=0$

Olur.

\section{TARTIŞMALAR VE SONUÇ (DISCUSSIONS AND CONCLUSION)}

(1) ve (2) denklemlerini ve bu denklemlerin çözüm integrallerini tekrar ele alalım. Tüm çözümler incelendiğinde diferansiyel denklemin mertebesi kadar keyfi sabit içeren bir kısım ve keyfi sabit içermeyen bir kısım olmak üzere iki kısımdan oluştuğu gözlenir. Bu denklemlerin keyfi sabit içeren kısmını $Y_{1}^{m}$ ile keyfi sabit içermeyen kısmını da $Y_{I I}^{m}$ ile belirtelim. Her bir genel çözüm $y^{m}=Y_{1}^{m}+Y_{\mathrm{II}}^{m}$ olarak yazılır. Buna göre, (1) ve (2) ile verilen 2. Yanlı diferansiyel denkleminin, homojen diferansiyel denkleminin genel çözümünün $Y_{\mathrm{II}}^{m} \mathrm{kısmı,} \mathrm{bir} \mathrm{özel}$ çözümünün de $Y_{1}^{m}$ kısmı olduğu gözlenir. $y^{(k)}=g(x)$ diferansiyel denklemini ele alalım. (1) denkleminde, $h=a \neq o$ ve $m=1$ veya $y^{m} \cdot h=Y$ alınırsa, benzer şekilde (2) de $h=0$ ve $m=1$ ya da $y^{m}+g=Y$ alınırsa, $y^{(k)}=g(x) \quad$ diferansiyel denklem tipi elde edilir. Böylece, üzerinde çalıştığımız k-kare diferansiyel denklemleri $y^{(k)}=g(x)$ diferansiyel denkleminden daha genel bir denklem olur. Benzer tartışmayı kompleks k-kare diferansiyel denklemler için de yaparız. 
Eğer (1) de $h$ fonksiyonu sırasıyla:

a) $h(x)=a_{k} x^{k}+a_{k-1} x^{k-1}+\cdots+a_{1} x+a_{0} \neq 0, a_{k} \neq 0$ polinomu alınırsa, $\left(y^{m} \cdot\left(a_{k} x^{k}+a_{k-1} x^{k-1}+\cdots+a_{1} x+a_{0}\right)\right)^{(k)}-g(x)=0$

Cauchy- Euler tipi diferansiyel denklemi elde edilir ve bu denklemin genel çözümü, Teorem $1^{\prime}$ den

$y^{m}=\frac{1}{a_{k} x^{k}+a_{k-1} x^{k-1}+\cdots+a_{1} x+a_{0}} \iiint \ldots \int g(x)(d x)^{k}$

olur.

b) $h(x)=a_{k}(b x+c)^{k}+a_{k-1}(b x+c)^{k-1}+\cdots+a_{1}(b x+c)+a_{0} \neq 0, a_{k} \neq 0, b \neq 0, c \neq 0$ polinomu alınırsa,

$\left(y^{m} \cdot\left(a_{k}(b x+c)^{k}+a_{k-1}(b x+c)^{k-1}+\cdots+a_{1}(b x+c)+a_{0}\right)\right)^{(k)}-g(x)=0$

Legendre tipi diferansiyel denklemi elde edilir ve bu denklemin genel çözümü, Teorem 1'den,

$y^{m}=\frac{1}{a_{k}(b x+c)^{k}+a_{k-1}(b x+c)^{k-1}+\cdots+a_{1}(b x+c)+a_{0}} \iiint \ldots \int g(x)(d x)^{k}$ olur.

(35) ve (37) diferansiyel denklemleri lineer olmayan denklemlerdir. Eğer bu denklemlerde $m=1$ alınırsa lineer tipleri olur. Süper pozisyon ilkesi için (1) denkleminde $g(x)=f_{1}(x)+f_{2}(x)+\cdots+f_{n}(x)$ alalım. Elde edilen denklem bir süperpozisyon denklemi olur. Bu elde edilen diferansiyel denkleminin genel çözümü ise, süperpozisyon ilkesini uygulama gereği duymadan, (3) gereğince

$$
y^{m}=\frac{1}{h(x)} \iiint \ldots \int\left(f_{1}(x)+f_{2}(x)+\cdots+f_{n}(x)\right)(d x)^{k}
$$

olur.

$$
\text { veya (4) gereğince, } \left.y^{m}=\iiint \ldots \quad \int\left(f_{1}(x)+f_{2}(x)+\cdots+f_{n}(x)\right) \quad(d x)^{k}-h(x)\right)
$$

Teorem 2 göz önüne alınarak tüm bunların kompleks değişkenli biçimi ifade edilebilir. Ayrıca Kesim 2'deki diğer Sonuçlar ve Kesim 3'deki Önermelerin kompleks değişkenli versiyonları yazılabilir

\section{8. ÇÖZÜMLÜ ÖRNEKLER (SOLVED EXEMPLES)}

$\mathrm{Bu}$ kesimde çalışmamıla ilgili bazı diferansiyel denklemleri ele alacağız ve bunların çözümünü verdiğimiz teoremler ışığında yapacağız.

Örnek 1. $y^{(5)}+15 y^{(4)}+90 y^{\prime \prime \prime}+270 y^{\prime \prime}+405 y^{\prime}+243 y=1 \quad$ diferansiyel denkleminin genel çözümü, $y=\frac{1}{243}+\left[C_{5}+C_{4} \frac{x^{4}}{4 !}+C_{3} \frac{x^{3}}{3 !}+C_{2} \frac{x^{2}}{2}+C_{1} x\right] \cdot e^{-3 x}$ dır . Çözüm: (15)' de $k=5$ ve $g(x)=h(x)=e^{3 x}$ alınır ve (16) uygulanır.

Örnek 2. $\mathrm{A} \neq 0, \cos a x \neq 0$ olmak üzere, $\cos a x \cdot y^{(5)}-5 \operatorname{asinax} \cdot y^{(4)}-10 a^{2} \cos a x \cdot y^{\prime \prime \prime}+10 a^{3} \operatorname{sinax} \cdot y^{\prime \prime}+5 a^{4} \cos a x \cdot y^{\prime}$

$$
-a^{5} \sin a x \cdot y=\cos a x
$$

diferansiyel denkleminin genel çözümü,

$y=\frac{1}{a^{5}} \cdot \operatorname{tanax}+\left[C_{5}+C_{4} \frac{x^{4}}{4 !}+C_{3} \frac{x^{3}}{3 !}+C_{2} \frac{x^{2}}{2}+C_{1} \cdot x\right] \cdot \frac{1}{\cos a x} \mathrm{~d} \mathrm{r}$.

Çözüm: (17) de $k=5, h(x)=g(x)=\operatorname{cosax}$ alalım.

$y=\frac{1}{\operatorname{cosax}} \iiint \iint \cos a x(d x)^{5} \quad$ eşitliği gereğince istenen çözüm bulunur.

Örnek 3. $e^{x} y^{\prime \prime}+2 e^{x} x y^{\prime}+e^{x} y=\ln x \quad(\ln x \neq 0)$ diferansiyel denkleminin genel çözümü

$y=\frac{1}{e^{x}} \cdot \iint \ln x(d x)^{2}=\frac{1}{2} x^{2} \cdot \frac{\ln x}{e^{x}}-\frac{3}{4} \cdot \frac{x^{2}}{e^{x}}+\left[C_{2}+C_{1} x\right] \cdot \frac{1}{e^{x}} \mathrm{~d} \mathrm{~d} r$.

Çözüm: (18) de $k=2, g(x)=e^{x}, h(x)=\ln x$ alırız.

Örnek 4. $x^{3} y^{\prime \prime \prime}+3 x^{2} y^{\prime \prime}+18 x y^{\prime}+6 y=\cos x, x \neq 0$ Cauchy-Euler diferansiyel denkleminin genel çözümü $\mathrm{y}=\frac{1}{x^{3}} \iiint \cos x(d x)^{3}=\frac{-\sin x}{x^{3}}+\left[C_{3}+C_{2} \frac{x^{2}}{2 !}+C_{1} x\right] \cdot \frac{1}{x^{3}}$ olur. 
Örnek 5. $(2 x+3)^{3} y^{\prime \prime \prime}+18(2 x+3)^{2} y^{\prime \prime}+72(2 x+3) y^{\prime}+48 y=\cos x$

diferansiyel denklemi bir Legendre tipidir. Bunun genel çözümü $y=\frac{1}{(2 x+3)^{3}} \iiint \cos x(d x)^{k}=\frac{-\sin x}{(2 x+3)^{3}}+\left[C_{3}+C_{2} \frac{x^{2}}{2 !}+C_{1} x\right] \cdot \frac{1}{(2 x+3)^{3}} \mathrm{~d} \_r$.

Örnek 6. (25) da $h(x)=\sin x \neq 0, g(x)=e^{x}, m=2$ alalım.

$2 \sin x \cdot y y^{\prime \prime \prime}+6 \sin x \cdot y^{\prime} y^{\prime \prime}+2 \cos x \cdot\left(y^{\prime}\right) 2-6 \sin x \cdot y y^{\prime}-\cos x \cdot y^{2}=e^{x}$ diferansiyel denkleminin genel çözümü $y^{2}=\frac{1}{\sin x} \iiint e^{x}(d x)^{3}=\frac{e^{x}}{\sin x}+\frac{1}{\sin x} \cdot\left[C_{3}+C_{2} \frac{x^{2}}{2 !}+C_{1} x\right]$ olur.

Örnek 7. (24) da $h(x)=\sin x \neq 0, g(x)=e^{x}, m=-2$ alalım. $-2 \sin x \cdot \frac{1}{y^{3}} y^{\prime \prime}+6 \sin x \cdot \frac{1}{y^{4}}\left(y^{\prime}\right)^{2}-4 \cos x \cdot \frac{1}{y^{3}} y^{\prime}-\sin x \cdot \frac{1}{y^{2}}=e^{x}$ diferansiyel denkleminin genel çözümü $y^{-2}=\frac{1}{\sin x} \iint e^{x}(d x)^{2}=\frac{e^{x}}{\sin x}+\frac{1}{\sin x}\left[C_{2}+C_{1} x\right]$ olur.

Örnek 8. (23)' de $h(x)=\sin x \neq 0, g(x)=e^{x}, m=\frac{4}{3}$ alalım. $\frac{4}{3} \sin x \cdot y^{\frac{1}{3}} y^{\prime}+\cos x \cdot y^{\frac{4}{3}}=e^{x}$ diferansiyel denkleminin genel çözümü $y^{\frac{4}{3}}=\frac{1}{\sin x} \int e^{x} d x=\frac{e^{x}}{\sin x}+\frac{C}{\sin x}$ olur.

Örnek 9. $p(x)=(2 x+3)^{3}+(2 x+3)^{2}+(2 x+3)+5 \neq 0$ polinomu için $\left((2 x+3)^{3}+(2 x+3)^{2}+(2 x+3)+5\right) y^{\prime \prime \prime}+\left(18(2 x+3)^{2}+24(2 x+3)+12\right) y^{\prime \prime}+$ $(72(2 x+3)+24) y^{\prime}+48 y=\cos x$

diferansiyel denklemi bir Legendre tipidir. Bu denklemin genel çözümü, $y=\frac{1}{(2 x+3)^{3}+(2 x+3)^{2}+(2 x+3)+5} \iiint \cos x(d x)^{k}=\frac{-\sin x}{(2 x+3)^{3}+(2 x+3)^{2}+(2 x+3)+5}+\left[C_{3}+C_{2} \frac{x^{2}}{2 !}+C_{1} x\right]$. $\frac{1}{(2 x+3)^{3}+(2 x+3)^{2}+(2 x+3)+5}$ olur.

Örnek $\quad 10 \quad . z \neq 0,5 z^{3} w^{\prime \prime \prime}+15 z^{2} w^{\prime \prime}+90 z w^{\prime}+30 w=\cos z$ kompleks diferansiyel denkleminin genel çözümü, $w=\frac{1}{5 z^{3}} \iiint \cos z(d z)^{3}=\frac{-\sin z}{5 z^{3}}+\left[C_{3}+C_{2} \frac{z^{2}}{2 !}+C_{1} z\right] \cdot \frac{1}{5 z^{3}}$ olur.

Örnek 11. $\quad p(z)=2 z^{3}+4 z^{2}+5 z+10 \neq 0$ polinomu alalım; $\left(2 z^{3}+4 z^{2}+5 z+10\right) w^{\prime \prime \prime}+\left(18 z^{2}+24 z\right) w^{\prime \prime}+(36 z+24) w^{\prime}+12 w=$ cos $\quad$ kompleks diferansiyel denkleminin genel çözümü,

$w=\frac{1}{2 z^{3}+4 z^{2}+5 z+10} \iiint \cos z(d z)^{3}=\frac{-\sin z}{2 z^{3}+4 z^{2}+5 z+10}+\left[C_{3}+C_{2} \frac{z^{2}}{2 !}+C_{1} z\right] \cdot \frac{1}{2 z^{3}+4 z^{2}+5 z+1 o}$ olur.

Örnek 12. $\sin 2 z \cdot w^{\prime \prime}+4 \cos 2 z \cdot w^{\prime}-\sin 2 z \cdot w=\sin 2 z+\cos 2 z-e^{z}+z \quad(\sin 2 z \neq 0)$ ya da, $\quad w^{\prime \prime}+4 \operatorname{cotan} 2 z \cdot w^{\prime}-w=1+\operatorname{cotan} 2 z-\frac{e^{z}}{\sin 2 z}+\frac{z}{\sin 2 z} \quad$ süper pozisyon kompleks diferansiyel denkleminin genel çözümü, $w=\frac{1}{\sin 2 z} \iint\left(\sin 2 z+\cos 2 z-e^{-z}+z\right)(d z)^{2}=\frac{1}{\sin 2 z}\left(-\frac{\sin 2 z}{4}-\frac{\cos 2 z}{4}-e^{-z}+\frac{z^{3}}{6}\right)+$ $\frac{1}{\sin 2 z}\left[C_{2}+C_{1} z\right]$ dir.

\section{KAYNAKLAR (REFERENCES)}

[1] Sveshnikov, A. and Tikhonov, A., (1978). The Theory of Functions of a Complex Variable. Translated from the Russian by George Yankovsky, Mır Publishers, Moskov

[2] Dettmann, J.W., (1965). Applied Complex Variables. The Macmillan Company, New York.

[3] Terziler, M., Öner, T. ve Öner, G., (2015). İleri Mühendislik Matematiği. Palme Yayıncılık, Ankara. (Tranlated from Russian by Erwin Kreyzig). 
[4] Piskinov, N., (1974). Differential and Integral Calculus. Vol:I, Translated from the Russian by George Yankovsky, Mır Publishers, Moskov.

[5] Churchill, R.V. and Brown, J.W., (1990). Complex Variable and Applications. McGraw-Hill Publishing Company, Singapore.

[6] Eren, S.. ve Razbonyalı, M., (2006). Diferansiyel Denklemler. T.C. Maltepe Üniv. Yayınları, No:28.

[7] Pala, Y., (2013). Modern Uygulamalı Diferansiyel Denklemler. Nobel Akademik Yayıncılık Eğitim Danışmanlık Tic. Ltd. Şti. Ankara.

[8] Sezer, M., (1995). Diferansiyel Denklemler-II ve Çözümlü Problemler. Baskı: Kanyılmaz Matbaacılık, İzmir.

[9] Çelik, A. ve Erduran, A., (2017). Bazı Belirsiz İntegraller İçin İndirgeme Formülleri ve İmproper İntegraller Üzerine. Pysical Sciences (NWSAPS), $12(4): 22-33$, DOI: $10.12739 /$ NWSA. 2017.12.4.3A0080. 